\title{
Education and training in optics conducted at Canon
}

\section{Kazuo Tanaka}

Kazuo Tanaka, "Education and training in optics conducted at Canon," Proc. SPIE 4588, Seventh International Conference on Education and Training in Optics and Photonics, (28 May 2002); doi: 10.1117/12.468680

SPIE Event: Education and Training in Optics and Photonics 2001, 2001, Singapore, Singapore 


\title{
Education and Training in Optics Conducted in CANON Kazuo TANAKA \\ Technology Administration Headquarters, CANON Inc. \\ 30-2, Shimomaruko 3-chome, Ohta-ku, Tokyo 146-8501, JAPAN \\ tanaka.kazuo205@canon.co.jp, User494704@aol.com
}

\begin{abstract}
The philosophy for human resources development in CANON Inc is based on the three-self spirit (Self-motivation, Self-awareness and Self-management). The Canon's R\&D engineers are required a positive attitude, creativity and courage to research and to develop products of innovation.

Educational measures in optics being in effect in Canon consist of the in-house training courses, studying abroad, publication and others.

\section{Introduction}

Canon is one of the creative companies in the world.

It researches, develops and manufactures sophisticated products. R\&D technologies have diversified along with the business diversification. Among them, optics is one of the core-technologies

society through technologies. Here Canon's optical products are enumerated; B/W copier, color copier, laser beam printer, image scanner, SLR camera, digital camera, lens shutter camera, camcoder, semiconductor production system, zoom lens in use of broadcasting, fundas camera, $\mathrm{X}$ ray camera and others.
\end{abstract} in Canon. People are the company's most valuable asset.

Continuing diversification of products and rapid progress of technologies require continued education in the company. Education and training are essential to all Canon people and they are vital for nurturing of the talents necessary for $R \& D$ activities.

In Canon, various education and training programs are provided.

\section{The compendium of Canon}

Canon's corporate philosophy is stated as "To be a global corporation providing kyosei (living and working together for the common goods) in all countries where we operate. Canon's corporate mission is to make a positive contribution through continued growth and reinvestment in the world's communities. Our objectives are as follows: We are responsible global citizens; We will create unique products of the highest quality based upon leading-edge technologies; and We will build an ideal company for continuing prosperity. Our values are characterized by the five statements: We respect cultural differences among ourselves, our customers and our communities; We believe that self-motivation, self-awareness and self-management are the three keys to good results; We respect the dignity, value the initiative and recognize the merit of each individual; and As members of the Canon family of companies, we trust and respect each other and work together harmoniously. Above all, we sustain our physical and emotional health in order to lead full and happy lives.

Canon has been a company which contributes to the

\section{The basic thinking of Canon R\&D engineers development}

1. Self-development is a basis of human resource development.

The basic thinking of "self-development" is common to all Canon R\&D engineers regardless of age and research themes. Therefore, saying "please train me" or "please teach me" is not regarded the right attitude at Canon. It is only from "active learning" instead of "passive teaching" that engineers can obtain good results. Canon R\&D engineers are asked to practice self-development according to the three-self spirit. That is to have a wish to improve weak points (self-awareness), to set own goals without being told (self-motivation), and to manage daily activities (self-management). Education cannot help a person if he/she has no motivation for learning and developing. In other words, the three-self spirit is the starting point of education at Canon.

\section{Workplace is the most important training room.}

We are qualified as professionals only when we apply our abilities and knowledge obtained through self-development fully to our work. The workplace where we can demonstrate our abilities is also the most important training room. Experiences obtained through daily work with seniors, colleagues, and subordinates are very valuable. Only able people are qualified as professionals. The workplace where such professionals exhibit their abilities is the most important training room.

3. Training opportunities must be planned and used by engineers themselves.

Now that technologies are diversified and innovated 
very quickly, only engineers who deal with the technologies can correctly recognize the trend. Training opportunities are not something that "are given" but rather, they should be "sought and created". Training opportunities planned by engineers who know the best are the key to good results.

4. Every $R \& D$ manager is responsible for as-many-as and as-quick-as possible development of subordinates.

R\&D managers, while carrying out their duties, are always close to their subordinates. They are in a position to supervise job performance and discover personalities, characters and aptitudes of their subordinates. Human resource development should be ideally tailor made according to each individual's different personality. R\&D managers training efforts reflect on their subordinates.

5. Develop corporate culture to encourage everyone to show one's ability to the full.

Canon's corporate culture is that every one can freely speak out, regardless of position, while paying due respect to each other's professional knowledge. Talented human resources are nurtured in energetic culture and environment.

\section{CANON R\&D Engineers}

- The foundation of original R\&D is developed from good human nature.

R\&D activities are characterized by the fact that engineers must work in an environment where both known and unknown subjects co-exist.

We desire our engineers to have courage to challenge unknown subjects, and passion and vitality to overcome failures. At the same time, we want them to have balanced human nature - being strict to themselves; modest and sincere to accept others' opinions.

1. Always maintain a positive attitude.

R\&D engineers create new things. Their sources of energy are passion and vitality.

2. Set own goal and challenge it with creativity and courage.

R\&D engineers, whether working for technology development or product development, must set own goal. It is even more so if the development is original and state-of-the-art.

3. Have deep professional technologies and wide field of vision.

Now that technologies are further diversified, it is becoming more difficult to develop superior products with technologies of one field alone.

4. Inter-"feel" products and technologies.

The strength of manufacturer's technologies is measured by its products released on the market. 5. Be highly sensitive to and have insight Into information.

We live in the information age. Our daily life is flooded with various information. $R \& D$ engineers must be sensitive to information and find our useful ones for their research work.

\section{Have a profit mind.}

Canon's pursuit of profit is justified when the earned profit is reinvested in its R\&D activities, because by offering superior products, Canon is contributing to the societies, to the countries and in large to the world.

7. Renovate not only technologies but also management style.

R\&D engineers, who are in the forefront of the rapid technological innovation, must be able to change the system of their R\&D activities to cope with new technologies.

8. Choose the present actions toward the future.

There are two types of conception to envision the future. One is "IN-OUT" type in which the future is envisioned from the present as a starting point. This is most commonly used to make plans. The other type is called "OUT-1N" in which the present is viewed from the future as a starting point. The "OUT-IN" conception is said to be effective for dealing with human beings or management.

\section{Have professional skills for management.}

R\&D engineers must have professional skills for management in order to effectively carry out duties. They should know "PERT" for schedule management, "VE" for cost reduction, "Design Review" for quality, reliability and safety, and other commonly used skills such as "experiment planning method". These skills are equally effective as long-time experience.

10. Have good communications about own work.

Now that technologies are being diversified and innovated rapidly, $R \& D$ engineers are required to work with other engineers or even with marketing people in a project team. Thus, engineers must be able to explain their thinking and work to other team members. Good communication skill is one of the important qualifications for $R \& D$ engineers.

\section{Education and training in optics in Canon}

R\&D based on an understanding of the world market from a global perspective is essential. Various training programs are provided to acquire technologies that are directly connected to this business strategy. They are offered to engineers of not only the R\&D section but also the production section, as well as affiliated companies. 


\section{Classes}

The objective of this measure is to study technologies at all levels, from fundamental to state-of-the-art through lectures, exercises, and experiments. It covers various fields, which include optics.

(1) Optics for the new-comers

Consisting of geometrical optics (paraxial theory, aberration theory, ray-tracing), physical optics (wave, polarization, interference, diffraction, LASER), image formation theory (OTF), lens design, optical fabrication and various optical instruments (imaging, illuminating, scanning).

In Japan, there are no researchers in universities who are specialized in "lens design." Therefore, new lens design members must acquire lens engineering after entering the company. This class contains not only lectures but also practical training in lens design and optical fabrication.

(2) Aberration theory

Consisting of the derivation of Seidel aberration the relation between the 3-rd order and the ray aberrations, and the introduction of aberration theory of decentered system.

(3) Optical instruments

Consisting of the study of optical configurations of various optical system, such as those of magnifier, telescope, camera and camera lens, copier, laser beam printer, stepper, medical equipment, TV and cine optics, projector and others.

(4) Optical thin film

Consisting of theory (design) and processing.

(5) Optical fabrication workshop

Consisting of practical trainings of fabrication; they are generating, polishing and lapping with abrasive material, cleaning, centering, coating, adhesive joint, and surface inspection.

(6) Digital image technology

Consisting of Fourier analysis, digital image processing (sampling, quantization, coding theory, compression), color management and standards (JPEG, MPEG and others).

(7) Imaging optics for non-optical engineers

Consisting of paraxial theory, ray-aberration and basic optical instruments.

(8)Electro-optics for non-optical engineers

Consisting of wave theory, interference, diffraction, application of LASER and holography.

\section{Engineer study abroad scheme}

This scheme sends Canon's engineers to graduate school in Europe and the USA for two years with the objective of acquiring cutting-edge technology, preparing engineers for globalizaton and constructing an international human network. This scheme has begun in 1984 .

Up to now, two engineers studied optics at the California Institute of Technology and the University of Arizona, five engineers researched into imaging science at the University College London, McGill University, Stanford University the University of Sydney and Carnegie Mellon Univiersy, and three engineers were engaged with the research of optical material at Princeton University, the University of Rochester and Max-Plank Institute. Among them, four engineers received Ph.D. degrees and several person earned M.S. degrees.

\section{Canon Research Report}

Among researches accomplished in Canon, those which are highly evaluated by outside professionals are published and are distributed to experts in and outside the company.

Up to now, fourteen papers have been published and the followings deal with themes related with optics.

No.1 "Study of erosion of polished surface of optical glasses" (in Japanese) by Dr.M.Mitusi, No.2 "The fifth order aberration theory for the practical use and its application in optical design" (in Japanese) by Dr.Y.Matsui, No.3 "Theory of zoom lenses" (in Japanese) by Dr.K.Yamaji, No.4 "High-sensitivity interferometory" (in Japanese) by Dr.K.Matsumoto, No.5 Helmholtz-Kirchhoff integral theorem in electromagnetic diffraction and propagation phenomena" by Dr.T.Suzuki, No.6 "Paraxial theory of mechanically compensated zoom lenses by means of Gaussian brackets" by Dr.K.Tanaka, No.8 "The spectral sensitization of photoconductivity for dye-sensitized CdS" (in Japanese) by Dr.H.Nakatsui, No.9 "A new bipolar imaging device" (in Japanese) by Dr.N.Tanaka, No.10 "Image quality improvement in laser beam printer" (in Japanese) by Dr.N.Kawamura and No.11 "Ultra-minumte optics in semiconductor industry" (in Japanese) by Dr.A.Suzuki.

\section{Concluding notes}

The importance of optics/photonics in Canon was first described. Then the philosophy of R\&D engineers in Canon was presented and necessary characters for the Canon's engineers were enumerated. Finally, various educational/training measures in optics conducted in Canon were introduced; they were classes, study abroad theme and publication.

\section{References}

K.Tanaka, Proc.SPIE vol.2525 (1995) 652-656. 